\title{
The fastest ball games from the viewpoint of science
}

\author{
by \\ Miran Kondrič ${ }^{1}$
}

Science in racket sports is a process, not just a theory. The main ambition of the three-day ITTF Sports Science and World Racket Sports Congresses held on 23 to 25 May 2015 in Suzhou was to discuss the actual priorities for improving scientific research among all four major racket sports. Of course, we also cannot forget the other racket sports that generally form part of physical education or, on the other side, very popular games in the area of sports for all.

All four major racket sports belong to the most popular sports in the world. Players range from youth, recreational players to world-class, elite ones. In reflection of this fact, scientific studies on racket sports are increasing. Researchers in every field of interest have performed studies in separate and individual areas such as physical training, physiology, psychology, medicine, dietetics, physics, engineering, etc. It is envisaged that this issue of the Journal of Human Kinetics contains papers that will eventually be regarded as a major source of knowledge and material for advancing racket sports science. We express our thanks to the International Table Tennis Federation, the Chinese Table Tennis Association, Soochow University, the Organising Committee of the World Table Tennis Championship, the Badminton World Federation, and the World Commission of Sports Science for hosting the 14th International Table Tennis Sports Science Congress and 5th World Racket Sports Congress. The Congress was organised by members of the Soochow University, the Chinese Table Tennis Association and members of the ITTF Sports Science and Medical Committee with the support of the International Table Tennis Federation, with almost 200 participants taking part in the three days of sessions in Suzhou.

The current issue of the Journal of Human Kinetics includes 15 papers from different areas of racket sports. Our hope is that this issue of the Journal together with the Congress Proceedings will contribute to the dissemination and enrichment of knowledge about racket sports. We are quite confident that those participating in these two congresses have taken home some of the exciting scientific experiences which the fastest ball games can provide. We also hope this and future publications will help acheve the fundamental goal of the International Federations; that is, to bridge the gap between sports scientists and practitioners regarding teaching, coaching, training and rehabilitation. We do not want people sitting around in committees all day and night; we want them out in sports halls and at training sessions, influencing players and making a difference to their performance.

Miran Kondrič

ITTF Sports Science and Medical Committee, Chairman

1 - ITTF Sports Science and Medical Committee, Chairman. 